\title{
Temperature-dependent optical properties of titanium nitride
}

\author{
Justin A. Briggs, ${ }^{1,2, a)}$ Gururaj V. Naik, ${ }^{3}$ Yang Zhao, ${ }^{2}$ Trevor A. Petach, ${ }^{4}$ \\ Kunal Sahasrabuddhe, ${ }^{1}$ David Goldhaber-Gordon, ${ }^{4}$ Nicholas A. Melosh, ${ }^{2}$ \\ and Jennifer A. Dionne ${ }^{2}$ \\ ${ }^{1}$ Department of Applied Physics, Stanford University, 348 Via Pueblo, Stanford, California 94305, USA \\ ${ }^{2}$ Department of Materials Science and Engineering, Stanford University, 496 Lomita Mall, Stanford, \\ California 94305, USA \\ ${ }^{3}$ Department of Electrical and Computer Engineering, Rice University, MS-378, Houston, Texas 77251-1892, \\ USA \\ ${ }^{4}$ Department of Physics, Stanford University, 382 Via Pueblo, Stanford, California 94305, USA
}

(Received 20 January 2017; accepted 19 February 2017; published online 6 March 2017)

\begin{abstract}
The refractory metal titanium nitride is promising for high-temperature nanophotonic and plasmonic applications, but its optical properties have not been studied at temperatures exceeding $400{ }^{\circ} \mathrm{C}$. Here, we perform in-situ high-temperature ellipsometry to quantify the permittivity of TiN films from room temperature to $1258^{\circ} \mathrm{C}$. We find that the material becomes more absorptive at higher temperatures but maintains its metallic character throughout visible and near infrared frequencies. X-ray diffraction, atomic force microscopy, and mass spectrometry confirm that TiN retains its bulk crystal quality and that thermal cycling increases the surface roughness, reduces the lattice constant, and reduces the carbon and oxygen contaminant concentrations. The changes in the optical properties of the material are highly reproducible upon repeated heating and cooling, and the room-temperature properties are fully recoverable after cooling. Using the measured hightemperature permittivity, we compute the emissivity, surface plasmon polariton propagation length, and two localized surface plasmon resonance figures of merit as functions of temperature. Our results indicate that titanium nitride is a viable plasmonic material throughout the full temperature range explored. Published by AIP Publishing. [http://dx.doi.org/10.1063/1.4977840]
\end{abstract}

High-temperature nanophotonics promises to impact technologies ranging from photo-thermal tumor ablation ${ }^{1}$ and plasmon-enhanced photocatalysis ${ }^{2}$ to heat-assisted magnetic recording $(\mathrm{HAMR})^{3}$ and thermophotovoltaics. ${ }^{4}$ Several of these applications demand temperatures exceeding $400{ }^{\circ} \mathrm{C},{ }^{3,5-7}$ a regime in which traditional plasmonic materials such as silver and gold exhibit pronounced degradation of their optical response. ${ }^{8}$ Accordingly, the refractory metal titanium nitride (TiN) has garnered considerable interest. $^{9,10}$ Indeed, with optical properties similar to gold ${ }^{11}$ and the possibility for fabrication via scalable processes, ${ }^{12}$ TiN is already being deployed in high-temperature plasmonics applications. ${ }^{13,14}$ However, in the critical range above $400{ }^{\circ} \mathrm{C}$, the temperature-dependent behavior of the permittivity of TiN was heretofore unknown. ${ }^{15}$

In this letter, we present the temperature-dependent optical properties of TiN from room temperature up to over $1200^{\circ} \mathrm{C}$. We find that at elevated temperatures, the real part of the permittivity becomes less negative, while the imaginary part increases, but the overall metallic character in the visible and near infrared (NIR) is retained. Further, we demonstrate the stability of the optical properties of this material by monitoring the permittivity while repeatedly cycling between $20^{\circ} \mathrm{C}$ and $1200^{\circ} \mathrm{C}$. The permittivity of the TiN film exhibits highly reproducible changes in the visible and NIR during cycling, and we observe a full recovery of the original room-temperature permittivity after heating and cooling. We

\footnotetext{
${ }^{\text {a) }}$ Author to whom correspondence should be addressed. Electronic mail: jabriggs@stanford.edu
}

then show how the surface morphology, crystal structure, and composition change as a result of this thermal cycling, demonstrating a material that is highly robust to elevated temperatures. Finally, we compute the emissivity, the surface plasmon polariton propagation length $\left(L_{S P P}\right)$, and two localized surface plasmon resonance (LSPR) figures of merit (FOMs) at temperatures between $20^{\circ} \mathrm{C}$ and $1258^{\circ} \mathrm{C}$. These calculations create a forecast for future device performance, indicating that TiN can serve as a material platform for visible and NIR devices operating at extreme temperatures.

We grow TiN films on nearly lattice-matched (100) magnesium oxide $(\mathrm{MgO})$ substrates using reactive DC magnetron sputtering. After growing a $\sim 225 \mathrm{~nm}$ thick film, we use spectroscopic ellipsometry to measure the room-temperature reflectance and then extract the relative permittivity using a combination of Drude and critical point oscillators 8,16

$$
\begin{aligned}
\epsilon(\omega)= & \epsilon_{b}-\frac{\omega_{p}^{2}}{\omega^{2}+i \gamma \omega} \\
& +\sum_{n=1}^{3} \frac{A_{n}}{\omega_{n}^{3 / 2} \chi_{n}^{2}}\left[2-\sqrt{1+\chi_{n}}-\sqrt{1-\chi_{n}}\right] .
\end{aligned}
$$

Here, $\epsilon_{b}$ is the background permittivity and $\omega_{p}$ and $\gamma$ are the plasma frequency and Drude damping; $\chi_{n}=\left(\omega+i \Gamma_{n}\right) / \omega_{n}$; and $A_{n}, \omega_{n}$, and $\Gamma_{n}$ are the critical point amplitude, center frequency, and damping.

To characterize the temperature-dependent optical response, we built a high-temperature ellipsometry chamber, fitted with optical ports, a turbo pump, and electrical feedthroughs to power a heater and measure the temperature 
via a thermocouple (Fig. S1, supplementary material). Understanding changes in permittivity between $20^{\circ} \mathrm{C}$ and $1200^{\circ} \mathrm{C}$ is critically important for many plasmonics applications, from HAMR transducers, which can experience temperatures up to $\sim 500^{\circ} \mathrm{C},{ }^{5,6}$ to thermophotovoltaics, which can require temperatures in excess of $1000{ }^{\circ} \mathrm{C}$ for high-efficiency operation. ${ }^{7}$ As such, we perform in-situ, high-temperature spectroscopic ellipsometry at a range of temperatures between $20^{\circ} \mathrm{C}$ and $1260^{\circ} \mathrm{C}$, at $\sim 1 \times 10^{-6}$ Torr. The resulting permittivity data are shown in Figs. 1(a) (real part) and 1(b) (imaginary part). As the temperature is increased, $\operatorname{Re}[\epsilon]$ becomes less negative throughout the visible and NIR, indicating a less metallic response. Simultaneously, $\operatorname{Im}[\epsilon]$ increases, indicating a lossier (i.e., more absorptive) film. In spite of these changes, TiN still exhibits a strong metallic response in the visible and NIR. Further, after cooling, the real and imaginary permittivities completely recover their original room-temperature values, as shown by the dotted lines in Fig. 1.

Technologies employing high-temperature plasmonic components will likely experience variation in temperature over time. As such, we assess the thermal durability of TiN via repeated thermal cycling between room temperature and $\sim 1200{ }^{\circ} \mathrm{C}$ over the course of $\sim 12 \mathrm{~h}$. The changes in the optical constants over these cycles are highly reproducible, and after cooling the permittivity always returns to its original room temperature values (Fig. S2, supplementary material). To quantitatively explore the behavior of $\epsilon(\omega, T)$, we examine the evolution of the screened plasma frequency $\left(\omega_{p}^{s}\right)$, the frequency at which the real permittivity crosses from positive to negative, signaling the onset of metallic behavior. In addition, we monitor the imaginary part of the permittivity at this frequency $\left(\operatorname{Im}\left[\epsilon\left(\omega_{p}^{s}\right)\right]\right)$ to quantify the loss at the metallic threshold.

Figures 2(a) and 2(b) show how these parameters evolve as a function of temperature over all four thermal cycles as we heat and cool the TiN film. Notably, the screened plasma frequency changes by only $\sim 5 \%$ and never drops below $2.5 \mathrm{eV}(\sim 500 \mathrm{~nm})$, demonstrating that TiN retains its metallic response in the visible regime even at temperatures exceeding $1200^{\circ} \mathrm{C}$. Meanwhile, $\operatorname{Im}\left[\epsilon\left(\omega_{p}^{s}\right)\right]$ at $1258^{\circ} \mathrm{C}$ is almost double its room temperature value but still retains a low upper bound of 4.5 .

The temperature evolution of the permittivity in the NIR is of utmost importance for many applications, especially thermal emission control for thermophotovoltaics. As such,
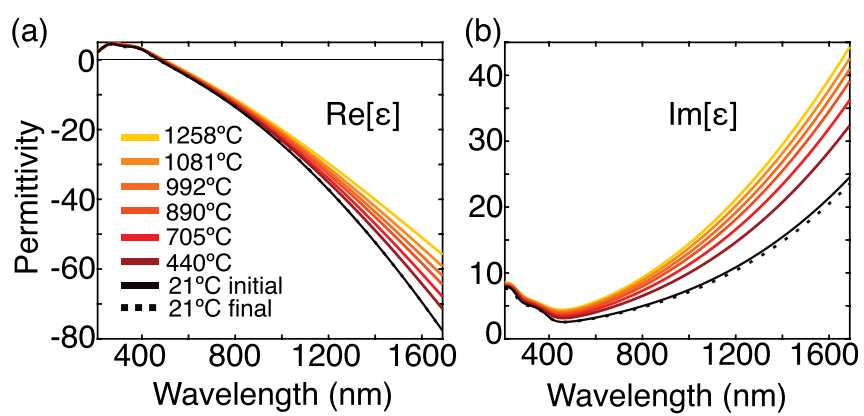

FIG. 1. Temperature-dependent optical properties of TiN. The real (a) and imaginary (b) parts of the relative permittivity of TiN as a function of temperature, from $21^{\circ} \mathrm{C}$ up to $1258^{\circ} \mathrm{C}$.

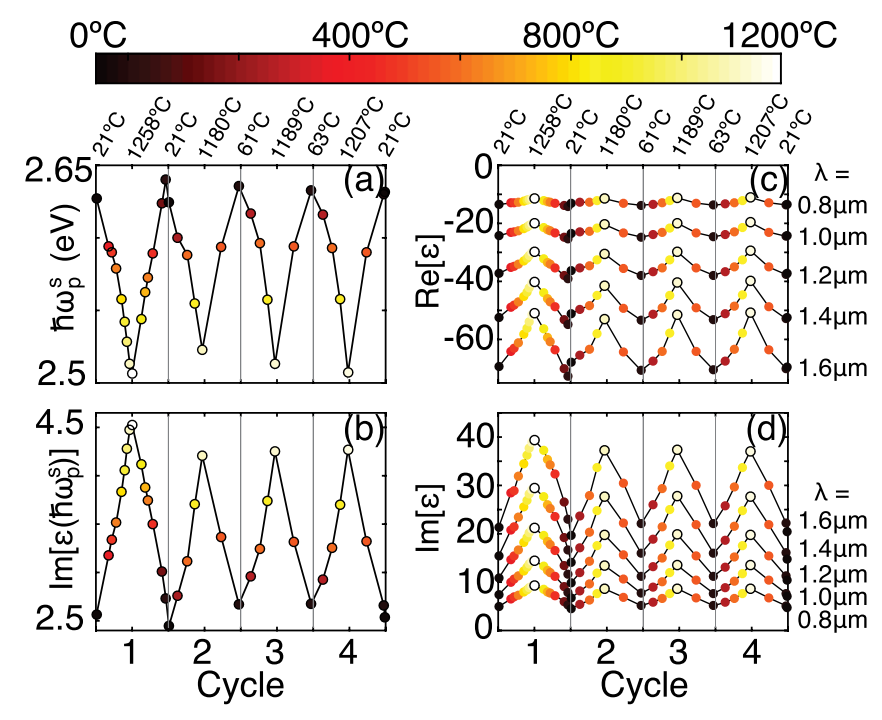

FIG. 2. Temperature evolution of TiN optical parameters with thermal cycling. The temperature dependence of the screened plasma frequency (a) and the imaginary component of the permittivity at the screened plasma frequency (b). The real part (c) and imaginary part (d) of the permittivity in the NIR, as a function of temperature. The measured temperature extrema for each cycle are listed on top of the plot, and the contrast of each data point is proportional to the temperature at which it was measured.

Figs. 2(c) and 2(d) plot the real and imaginary components, respectively, of the permittivity at numerous NIR wavelengths, as a function of temperature, for all thermal cycles. Again, we see that the changes in $\epsilon$ are highly reproducible and the room temperature values are fully recoverable. As the wavelength is increased, the magnitude of the change in the real and imaginary permittivities becomes larger. However, $\operatorname{Re}[\epsilon]$ remains well below zero for all frequencies studied here, demonstrating that TiN can support applications requiring a high-temperature metal in the NIR.

These trends can be understood by considering two predominant underlying physical changes that occur with heating: increased phonon population and volume expansion. Higher temperatures imply greater phonon scattering, which leads to more absorption and a larger $\operatorname{Im}[\epsilon]$, as observed. Simultaneously, as rising temperatures increase the volume of the TiN film, both the carrier concentration $n$ and the screened plasma frequency-proportional to $\sqrt{n}$-are reduced. If volume expansion were the only mechanism at play, we would expect a $\sim 1.7 \%$ decrease in the screened plasma frequency; however, we experimentally observe a $\sim 4.6 \%$ decrease, indicating the presence of other effects. Indeed, we also observe broadening of the interband transitions in the visible regime with increased temperature (Fig. S3, supplementary material). This is qualitatively consistent with the larger observed decrease in screened plasma frequency, ${ }^{17}$ but quantitative modeling of this effect is beyond the scope of this work.

Now that we have seen how the optical properties of TiN evolve at high temperatures, we would like to understand if any permanent material changes occur as a result of exposure to high temperature. We start by using atomic force microscopy (AFM) to visualize the TiN surface morphology before and after repeated thermal cycling between room temperature and $\sim 1200^{\circ} \mathrm{C}$, as seen in Figs. 3(a) and 3(b). Before 


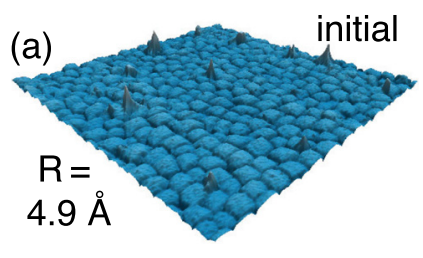

$0 \quad$ Height $(\mathrm{nm}) \quad 20$

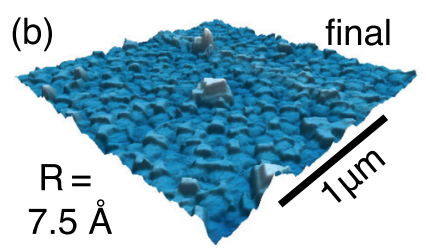

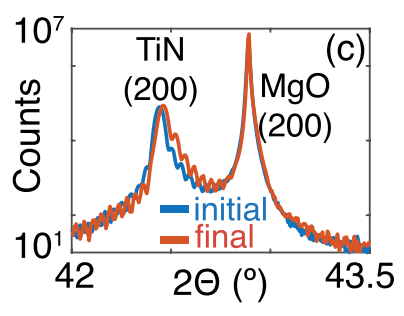

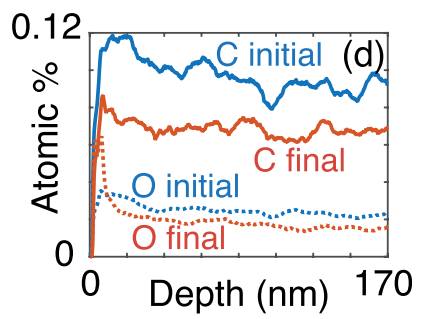

FIG. 3. Material characterization of TiN before ("initial") and after ("final") repeated thermal cycling up to $\sim 1200^{\circ} \mathrm{C}$. AFM showing the surface morphology before (a) and after (b) four heating/cooling cycles. The root mean square roughness increases from $\mathrm{R}=4.9 \AA$ to $\mathrm{R}=7.5 \AA$ after thermal cycling. (c) X-ray diffraction patterns (symmetric scans) of TiN before and after heating, with the (200) peaks of the TiN film and the $\mathrm{MgO}$ substrate visible. (d) Secondary ion mass spectrometry depth profiles showing the concentration of contaminant carbon and oxygen in $\mathrm{TiN}$ as a function of depth, before and after heating.

heating, the film surface is nearly atomically smooth, with a root mean square surface roughness of $R \sim 4.93 \AA$ (TiN's room-temperature lattice constant is $4.24 \AA$ ). ${ }^{18}$ A mosaic pattern and the presence of pronounced pyramidal structures respectively indicate columnar grains and periodic strain relaxation due to the small $(0.71 \%)$ mismatch between the TiN and $\mathrm{MgO}$ lattice constants. After thermal cycling, the TiN surface exhibits notable reconfiguration and an increase in roughness from $\sim 4.93 \AA$ to $\sim 7.51 \AA$.

To determine if these surface changes are concomitant with a change in the bulk film structure, we use x-ray diffraction (XRD) to assess the TiN crystal structure before and after heating, as shown in Fig. 3(c). In a symmetric scan, the asdeposited film (i.e., before heating, labeled "initial" in the figure) exhibits a (200) TiN peak (at slightly lower angle than the (200) $\mathrm{MgO}$ substrate peak) flanked by finite-thickness fringes. Combined with an absence of any peaks in the grazing incidence scan (Fig. S4, supplementary material) and the mosaic surface structure illuminated by the AFM map in Fig. 3(a), these data suggest columnar epitaxy of TiN on MgO. After thermal cycling, a symmetric XRD scan reveals that the orientation of the $\mathrm{TiN}$ film is retained (again, there is an absence of any peaks in the grazing incidence scan, Fig. S4, supplementary material). However, the TiN (200) peak has shifted to a slightly higher angle, suggesting a $\sim 0.04 \%$ decrease in the lattice constant along the (100) direction.

To understand the origin of this change in the lattice constant, we explore the composition of the TiN films as a function of depth using secondary ion mass spectrometry (SIMS). As depicted in Fig. 3(d), a depth profile of the as-deposited film indicates $0.094 \%$ carbon and $0.024 \%$ oxygen in the bulk of the film. Interestingly, after exposure to repeated thermal cycling, the film exhibits smaller contaminant concentrations of $0.068 \%$ carbon and $0.017 \%$ oxygen. This reduction in contamination is consistent with the reduction in the TiN lattice constant, assuming that the unwanted carbon and oxygen

atoms occupy interstitial sites in the TiN structure and thus serve to expand the lattice. The presence of strain relaxation at elevated temperatures is also consistent with our observations and could further explain the reduction in the TiN lattice constant resulting from thermal cycling.

This suite of optical and material characterization indicates that TiN holds great promise for high-temperature nanophotonics. For example, we have observed that the TiN loss roughly doubles when the temperature is increased from $20^{\circ} \mathrm{C}$ to over $1200^{\circ} \mathrm{C}$. In contrast, the loss in gold approximately doubles upon heating to only $500{ }^{\circ} \mathrm{C} .{ }^{8}$ Further, TiN is robust to high-temperature operation, exhibiting neither permanent permittivity degradation nor detrimental changes to the composition and structure. This is again in contrast to traditional plasmonic materials that suffer significant and permanent degradation in optical properties and material structure after heating to only half the temperature we have explored here. ${ }^{8}$ Thus, TiN is a particularly promising material for applications demanding exposure to high temperatures.

To explore how the functionality of TiN-based devices will change during high-temperature operation, we use our measured temperature-dependent permittivity data to compute the emissivity, $L_{S P P}$, and two LSPR figures of merit, as functions of temperature.

The temperature-dependent emissivity, computed for an optically thick planar TiN film using the transmission matrix method, is shown in Fig. 4(a). At room temperature, the emissivity peaks at 0.86 in the visible regime due to strong interband absorption in the TiN film. The onset of metallic behavior is signaled by the rapid drop-off in the emissivity at $\sim 500 \mathrm{~nm}$, giving way to a regime of highly suppressed emissivity in the NIR. As the temperature is increased, the emissivity peak is broadened and damped to 0.78. Simultaneously, the emissivity cutoff at $\sim 500 \mathrm{~nm}$ becomes less sharp and the suppression in the NIR less complete. For applications relying on visible and NIR emissivity control, such as selective thermal emitters for thermophotovoltaic energy conversion, this heightened NIR emissivity at high temperatures will result in reduced efficiency. For example, in going from room temperature to $1200{ }^{\circ} \mathrm{C}$, the emissivity at $1600 \mathrm{~nm}$ increases from 0.07 to 0.15 . However, the fact that the NIR emissivity remains below 0.20 at over $1200{ }^{\circ} \mathrm{C}$ bodes well for hightemperature thermal emission control applications; for example, tantalum photonic crystal selective thermal emitters exhibit IR emissivities of $\sim 0.10$ at room temperature and $\sim 0.25$ at $1200{ }^{\circ} \mathrm{C}$.

To understand how applications utilizing surface plasmon polaritons (SPPs) will be impacted by high-temperatures, we compute the SPP propagation length at an air-TiN interface as $L_{S P P}=(2 * \operatorname{Im}[\beta])^{-1}$, where $\beta$ is the SPP propagation vector. ${ }^{19}$ As depicted in Fig. 4(b), the propagation length is reduced as temperature, and thus absorption in the metal, increases. At $1600 \mathrm{~nm}, L_{S P P}$ is reduced from $62 \mu \mathrm{m}$ to $26 \mu \mathrm{m}$ upon heating from room temperature to $1258^{\circ} \mathrm{C}$. This reduction in $L_{S P P}$ is of paramount importance for devices relying on SPP waveguiding. For example, both the sensitivity of SPP-based biosensors ${ }^{20}$ and the functionality of plasmonic integrated circuit interconnects ${ }^{21}$ depend critically on SPP propagation length. 

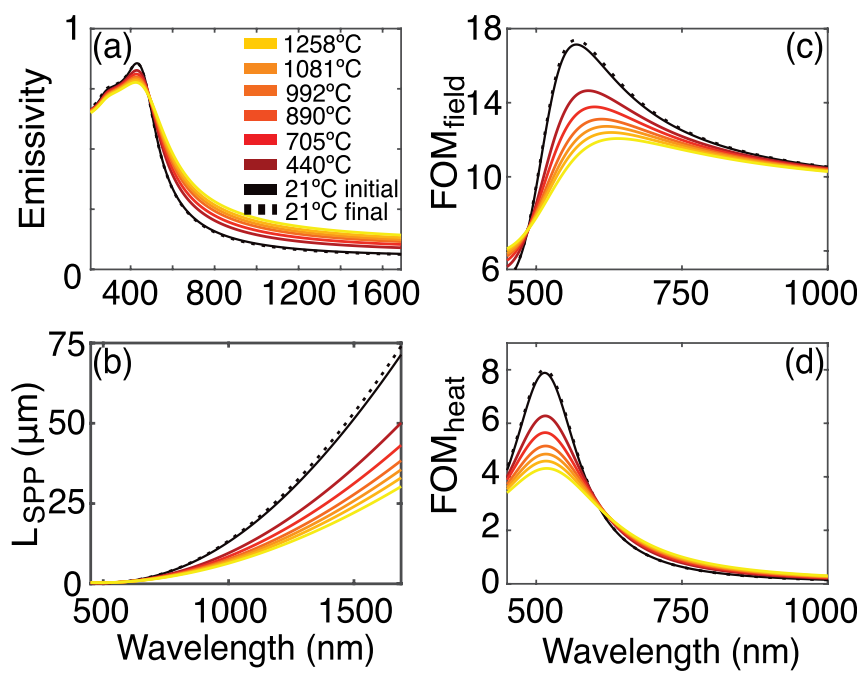

FIG. 4. Application-relevant optical parameters of TiN as a function of temperature. (a) Emissivity of an optically thick TiN film, calculated via the T-matrix method. (b) The surface plasmon polariton propagation length, $(2 \times \operatorname{Im}[\beta])^{-1}$, for an air-TiN interface. (c) The field intensity enhancement figure of merit, $F O M_{\text {field }}=\left|E_{\max } / E_{o}\right|^{2}$, and (d) the heat generation figure of merit, $F O M_{\text {heat }}=\left(\hbar \omega * \operatorname{Im}[\epsilon] / n_{s}\right) *\left|E_{\text {in }} / E_{o}\right|^{2}$, for a spherical TiN nanoparticle in vacuum.

A nanoantenna is the basis for many plasmonics applications, ranging from heat-assisted magnetic recording and biosensing to photo-thermal therapy and imaging. To understand how the functionality of these and other resonator-based devices will change at elevated temperatures, we compute two figures of merit (FOMs) for spherical TiN nanoparticles, as functions of temperature. The electric field intensity enhancement FOM (given as $F O M_{\text {field }}=\left|E_{\max } / E_{o}\right|^{2}$, where $E_{\text {max }}$ is the maximum electric field outside the particle and $E_{o}$ is the incident field) is relevant for LSPR applications in which field enhancement is desirable; likewise, the heat generation FOM (given as $F O M_{\text {heat }}=\left[\hbar \omega * \operatorname{Im}[\epsilon] / n_{s}\right] *\left|E_{\text {in }} / E_{o}\right|^{2}$, where $E_{\text {in }}$ is the electric field inside the particle and $n_{s}$ is the index of the surrounding medium) is relevant for applications in which localized heat production is desirable. ${ }^{22}$ Figures $4(\mathrm{c})$ and $4(\mathrm{~d})$ respectively plot these two FOMs for TiN nanoparticles in vacuum (see supplementary material for the traditional LSPR FOM, $-\operatorname{Re}[\epsilon] / \operatorname{Im}[\epsilon])$. The room-temperature field intensity enhancement FOM peaks at $\sim 17$ near $570 \mathrm{~nm}$ and is damped to $\sim 12$ and red-shifted to $640 \mathrm{~nm}$ at $1258^{\circ} \mathrm{C}$. The peak in the heat generation FOM is also damped at high temperatures, from $\sim 8$ at room temperature to just above 4 at $1258^{\circ} \mathrm{C}$. However, this damping is not accompanied by a redshift; rather, the peak remains at $\sim 515 \mathrm{~nm}$ at all temperatures. The evolution of these FOMs indicates that TiN-based technologies will experience reduced performance at elevated temperatures but that substantial field enhancement and heat generation can still be achieved. ${ }^{22}$

We have shown how the permittivity of a titanium nitride film evolves as a function of temperature, from $20^{\circ} \mathrm{C}$ to over $1200^{\circ} \mathrm{C}$. At these extreme temperatures, TiN becomes less metallic (smaller $-\operatorname{Re}[\epsilon]$ ) and more lossy (larger $\operatorname{Im}[\epsilon]$ ). These changes are highly reproducible and do not fundamentally alter the metallic character of the material. Additionally, the original room-temperature optical constants are fully recoverable upon cooling. Crystal structure, surface morphology, and composition studies before and after repeated thermal cycling indicate that TiN is robust to prolonged cyclic high-temperature operation. Finally, calculation of application-relevant optical parameters using our measured temperature-dependent permittivity data indicates that $\mathrm{TiN}$ will function as a viable plasmonic material, even in applications demanding a metallic response up to over $1200^{\circ} \mathrm{C}$. In all, this study suggests that titanium nitride can act as a robust material platform for high-temperature plasmonics applications ranging from cancer therapeutics to renewable energy generation.

See supplementary material for (1) sample fabrication details; (2) high-temperature ellipsometry experiment details; (3) a figure and table of all permittivity, sample temperature, and chamber pressure data for all four thermal cycles; (4) the temperature-dependent permittivity data in the interband transition regime; (5) details on the Drude + critical points modeling; (6) details on the AFM measurements and high-resolution AFM topographical maps; (7) details on the XRD measurements, grazing incidence XRD scans, and inference of the TiN lattice constant; (8) details on the SIMS measurements; (9) optical images of the TiN films before and after thermal cycling; (10) the traditional LSPR FOM as a function of temperature; and (11) raw reflectivity data taken on a room-temperature sample both inside and outside the chamber, showing that the optical ports do not affect the optical measurements.

J.A.B. would like to thank everyone at HeatWave Labs. J.A.B. was supported by NSF GRF (2012122469). This work is part of the "Light-Material Interactions in Energy Conversion" Energy Frontier Research Center under U.S. DOE, Office of Science, Office of Basic Energy Sciences award DE-SC0001293. X-ray studies were supported under DOE LDRD award DE-AC02-76F00515. Part of this work was performed at Stanford's SNSF and SNF.

${ }^{1}$ D. P. O’Neal, L. R. Hirsch, N. J. Halas, J. D. Payne, and J. L. West, "Photo-thermal tumor ablation in mice using near infrared-absorbing nanoparticles," Cancer Lett. 209, 171-176 (2004).

${ }^{2}$ P. Christopher, H. Xin, A. Marimuthu, and S. Linic, "Singular characteristics and unique chemical bond activation mechanisms of photocatalytic reactions on plasmonic nanostructures," Nat. Mater. 11, 1044-1050 (2012).

${ }^{3}$ W. A. Challener, C. Peng, A. V. Itagi, D. Karns, and W. Peng, "Heatassisted magnetic recording by a near-field transducer with efficient optical energy transfer," Nature Photonics 3, 220-224 (2009).

${ }^{4}$ V. Rinnerbauer, Y. X. Yeng, W. R. Chan, J. J. Senkevich, J. D. Joannopoulos, M. Soljačić, and I. Celanovic, "High-temperature stability and selective thermal emission of polycrystalline tantalum photonic crystals," Opt. Exp. 21, 11482-11491 (2013).

${ }^{5}$ N. Zhou, X. Xu, A. T. Hammack, B. C. Stipe, K. Gao, W. Scholz, and E. C. Gage, "Plasmonic near-field transducer for heat-assisted magnetic recording," Nanophotonics 3, 141-155 (2014).

${ }^{6}$ M. H. Kryder, E. C. Gage, T. W. McDaniel, W. A. Challener, R. E. Rottmayer, G. Ju, Y.-T. Hsia, and M. F. Erden, "Heat assisted magnetic recording," Proc. IEEE. 96, 1810-1835 (2008).

${ }^{7}$ N.-P. Harder and P. Würfel, "Theoretical limits of thermophotovoltaic solar energy conversion," Semicond. Sci. Technol. 18, S151-S157 (2003).

${ }^{8}$ H. Reddy, U. Guler, A. V. Kildishev, A. Boltasseva, and V. M. Shalaev, "Temperature-dependent optical properties of gold thin films," Opt. Mater. Express 9, 2776-2802 (2016).

${ }^{9}$ U. Guler, A. Boltasseva, and V. M. Shalaev, "Refractory plasmonics," Science 344, 263-264 (2014). 
${ }^{10}$ G. V. Naik, V. M. Shalaev, and A. Boltasseva, "Alternative plasmonic materials: Beyond gold and silver," Adv. Mater. 20, 3264-3294 (2013).

${ }^{11}$ G. V. Naik, B. Saha, J. Liu, S. M. Saber, E. A. Stach, J. M. K. Irudayaraj, T. D. Sands, V. M. Shalaev, and A. Boltasseva, "Epitaxial superlattices with titanium nitride as a plasmonic component for optical hyperbolic metamaterials," Proc. Natl. Acad. Sci. 111, 7546-7551 (2014).

${ }^{12}$ J. A. Briggs, G. V. Naik, T. A. Petach, B. K. Baum, D. Goldhaber-Gordon, and J. A. Dionne, "Fully CMOS-compatible titanium nitride nanoantennas," Appl. Phys. Lett. 108, 051110-1-051110-5 (2016).

${ }^{13}$ W. Li, U. Guler, N. Kinsey, G. V. Naik, A. Boltasseva, J. Guan, V. M. Shalaev, and A. V. Kildishev, "Refractory plasmonics with titanium nitride: Broadband metamaterial absorber," Adv. Mater. 26, 7959-7965 (2014).

${ }^{14}$ J. Liu, U. Guler, A. Lagutchev, A. Kildishev, O. Malis, A. Boltasseva, and V. M. Shalaev, "Quasi-coherent thermal emitter based on refractory plasmonic materials," Opt. Mater. Express 5, 2721-2728 (2015).

${ }^{15}$ S. Tripura Sundari, R. Ramaseshan, F. Jose, S. Dash, and A. K. Tyagi, "Investigation of temperature dependent dielectric constant of a sputtered TiN thin film by spectroscopic ellipsometry," J. Appl. Phys. 115, 0335161- 033516-6 (2014).
${ }^{16}$ WVASE Operational Maunal (J.A. Woollam Co., 2012).

${ }^{17}$ S. Logothetidis, J. Petalas, M. Cardona, and T. Moustakas, "Optical properties and temperature dependence of the interband transitions of cubic and hexagonal GaN," Physical Review B 50, 18017-18129 (1994).

${ }^{18}$ K. Aigner, W. Lengauer, D. Rafaja, and P. Ettmayer, "Lattice parameters and thermal expansion of $\operatorname{Ti}\left(\mathrm{C}_{x} \mathrm{~N}_{1-x}\right), \operatorname{Zr}\left(\mathrm{C}_{x} \mathrm{~N}_{1-x}\right), \operatorname{Hf}\left(\mathrm{C}_{x} \mathrm{~N}_{1-x}\right)$ and $\mathrm{TiN}_{1-x}$ from 298 to $1473 \mathrm{~K}$ as investigated by high-temperature $\mathrm{X}$-ray diffraction," J. Alloys Compd. 215, 121-126 (1994).

${ }^{19}$ L. Novotny and B. Hecht, Principles of Nano-Optics (Cambridge University Press, 2012).

${ }^{20}$ W. R. Wong, O. Krupin, F. R. Mahamd Adikan, and P. Berini, "Optimization of long-range surface plasmon waveguides for attenuationbased biosensing," J. Lightwave Technol. 33, 3234-3242 (2015).

${ }^{21}$ N. Kinsey, M. Ferrera, G. V. Naik, V. E. Babicheva, V. M. Shalaev, and A. Boltasseva, "Experimental demonstration of titanium nitride plasmonic interconnects," Opt. Express 22, 12238-12247 (2014).

${ }^{22}$ A. Lalisse, G. Tessier, and J. Plain, "Quantifying the efficiency of plasmonic materials for near-field enhancement and photothermal conversion," J. Phys. Chem. C 119, 25518-25528 (2015). 